\section{P4.46 INTRODUCTION OF RAPID SYPHILIS AND HIV TESTING IN PRENATAL CARE IN COLOMBIA: QUALITATIVE ANALYSIS}

${ }^{1}$ Hernando G Gaitan Duarte, ${ }^{1}$ Maria Teresa Ochoa-Manjarres, ${ }^{2}$ Sidia Caicedo, ${ }^{3}$ Berta Gomez, ${ }^{4}$ Freddy Perez. ${ }^{1}$ Universidad Nacional de Colombia, Bogota D.C. - Colombia; ${ }^{2}$ Ministerio de Salud y La Protección Social, Bogota D.C. - Colombia; ${ }^{3}$ Pan American Health Organisation, Bogota D.C. - Colombia; ${ }^{4}$ Pan American Health Organisation, Washington, D.C, USA

\subsection{6/sextrans-2017-053264.543}

Introduction Interpret perceptions of Colombian health professionals concerning factors that obstruct and facilitate the introduction of rapid syphilis and HIV testing in prenatal care services.

Methods A qualitative study based on semi-structured interviews was carried out. A convenience sample was selected with 37 participants, who included health professionals involved in prenatal care services, programs for pregnant women, clinical laboratories, and directors of health care units or centres, as well as representatives from regional departments and the Ministry of Health.

Results Colombia does not do widespread screening with rapid syphilis and HIV tests in prenatal care. The professionals interviewed stated they did not have prior experience in the use of rapid tests-except for laboratory staff-or in the course of action in response to a positive result. The insurance system hinders access to timely diagnosis and treatment. Health authorities perceive a need to review existing standards, strengthen the first level of care, and promote comprehensive prenatal care starting with contracts between insurers and health service institutional providers. Participants recommended staff training and integration between health-policymaking and academic entities for updating training programs Conclusion The market approach and the characteristics of the Colombian health system constitute the main barriers to implementation of rapid testing as a strategy for elimination of mother-to-child transmission of syphilis and HIV. Measures identified include making changes in contracts between insurers and health service institutional providers, adapting the timing and duration of prenatal care procedures, and training physicians and nurses involved in prenatal care.

\section{P4.47 MODELLING THE IMPACT OF A HYPOTHETICAL VACCINE THAT PREVENTS HSV-2 BUT INCREASES SUSCEPTIBILITY TO HIV}

Ian Spicknall, Harrel Chesson. Centres for Disease Control and Prevention, Atlanta, GA, USA

\subsection{6/sextrans-2017-053264.544}

Introduction An HSV-2 vaccine could reduce the health and economic burden of HSV-2, including neonatal herpes. However, such a vaccine might increase susceptibility to HIV infection through immune cell recruitment to genital tissues. If so, HSV-2 vaccination may decrease HSV-2 incidence but also increase HIV incidence.

Methods We explored these tradeoffs in a mathematical model of HSV-2 transmission dynamics and vaccination that includes static HIV incidence using ordinary differential equations. We modelled scenarios resembling the United States in terms of HSV-2 and HIV transmission. We examined scenarios where HSV-2 vaccination either did or did not increase HIV susceptibility. We assumed that HSV-2 infection led to increased HIV susceptibility, but no other interactions between HSV-2 and HIV.

Results Assuming vaccine efficacy against infectiousness of 0.5 , vaccination may decrease HSV-2 incidence by $22 \%$. However, when vaccination increases HIV susceptibility, this benefit comes at the cost of increased HIV incidence: 1.6, 2.0, or $2.5 \%$ in populations with low, moderate, and high HIV exposure rates, respectively. When vaccination does not increase HIV susceptibility, HIV incidence decreased by 7.0, 6.1, and $4.9 \%$, respectively. The magnitude of these effects depend on additional factors such as the degree to which HSV-2 vaccination increases HIV susceptibility, HSV-2 vaccine coverage, and baseline HSV-2 prevalence.

Conclusion Our analysis shows how models can describe tradeoffs of public health interventions offering substantial benefits and possible harms. Models can be used to weigh the relative benefits and costs of a specific intervention, or to examine whether an intervention results in a net gain or net loss in quality adjusted life years. Our analysis illustrates the need for updated estimates of the quality-of-life impact of HSV-2 in different individuals in order to weigh the public health benefits of HSV-2 vaccination against potentially adverse outcomes. More research is needed to better describe the interaction between HSV-2 and HIV infection and transmission.

\section{P4.48 PERCEPTION OF SOCIAL SUPPORT BETWEEN PEOPLE AND PEOPLE WITH HIV}

Jacqueline Silveira de Quadros, Stela Maris de Mello Padoin,Cristiane Cardoso de Paula. Universidade Federal de Santa Maria, Santa Maria - RS, Brazil

\subsection{6/sextrans-2017-053264.545}

Introduction Women living with Human Immunodeficiency Virus (HIV) are faced with personal, family and social difficulties, which can lead to non-compliance with measures to prevent vertical HIV transmission. One strategy in coping with the disease is the social support that plays a role in mitigating the negative effects of stressful events related to infection. The objective was to evaluate if the social support perceived by pregnant women is different from that perceived by puerperal women living with HIV.

Method This is a cross-sectional survey carried out in the municipality of Santa Maria - RS, Brazil. We used an instrument composed of a characterisation questionnaire; Social Support Scale for People Living with HIV/AIDS. The data collection was from April to November 2014, with 78 pregnant and postpartum women. Descriptive statistics, Student's T or Mann-Whitney test and linear logistic regression were used for the analysis of the data. The level of significance was considered with values equal or less than $5 \%$.

Results It was observed that being a pregnant woman is a protection factor when compared to being puerperal, being the chance of protection equal to 8840 times (CI: 1,012-16,767) for general social support, and 6010 times (IC: 1,532-10,489) for support Social instrumental.

Conclusion Health professionals need to know and interact with the social support network of these women, in order to implement actions that help health care during this period of family reorganisation. In addition, it is necessary to expand humanised attention and strengthen public policies. 